LBL-35940

\title{
Resonant Generation of Nonequilibrium Phonons in GaAs/AlAs Quantum Wells via Intersubband Scattering
}

\author{
Z.P. Su, T. Ruf, K.R. Wald, and P.Y. Yu \\ Department of Physics \\ University of California \\ and \\ Materials Sciences Division \\ Lawrence Berkeley Laboratory \\ University of California \\ Berkeley, California 94720
}

\section{K.T. Chan}

Chinese University of Hong Kong, Hong Kong

July 1994

This work was supported by the Director, Office of Energy Research, Office of Basic Energy Sciences, Materials Sciences Division, of the U.S. Department of Energy under Contract No. DE-AC03-76SF00098. 
To be presented at the 22nd Internat. Conf. on Physics of Semiconductors,

Vancouver, Canda, Aug. 15-19, 1994.

\title{
RESONANT GENERATION OF NONEQUILIBRIUM PHONONS IN GaAs/AIAs QUANTUM WELLS VLA INTERSUBBAND SCATTERING
}

\author{
Z.P. SU, T. RUF', K.R. WALD, P.Y. YU \\ Department of Physics, University of Califormia at Berkeley, Berkeley, CA94720 i.dd \\ Materials Sciences Division, Lawrence Berkeley Laboratory, Berkeley, CA94720
}

\author{
and \\ K.T. CHAN \\ Chinese University of Hong Kong, Hong Kong
}

\begin{abstract}
We have studied the resonant intersubband scattering of optical phonons or RISOP theoretically and by picosecond Raman scattering. Experimentally, we have established a way to determine phonon occupancy in quantum wells. Our model calculation shows that a significant amount of long wavelength LO phonons are generated by RISOP in GaAs/AlAs quantum wells. However, we measured a much smaller phonon population than predicted. The discrepancy can be explained by a breakdown of in-plane wavevector conservation due to intsrface roughness.
\end{abstract}

\section{Introduction}

When energetic electrons are pholoexcited by picosecond lasers into the conduction subbands of semiconductor quantum wells (QW), they relax by emitting nonequilibrium phonons via intrasubband and intersubband scattering. During these processes, both energy and $q_{/}$, wavevector parallel to the QW layers, have to be conserved. As a result, phonons generated by intrasubband scattering typically have $q_{/ /}$ much larger than $10^{5} \mathrm{~cm}^{-1}$, and, in principle, cannot be observed in Raman backscattering experiments due to the conservation of $q_{/ /}$in Raman processes. On the other hand, it has been suggested that, when the subband separation is close to one LO phonon energy, resonant intersubband scattering of optical phonons or RISOP will become important ${ }^{1}$. Since RISOP can produce phonons with wavevectors less than $10^{5} \mathrm{~cm}^{-1}$, Raman scattering is an ideal probe for studying RISOP. In this work, we report our theoretical and experimental study of RISOP using Raman scattering with picosecond tunable laser pulses. Our results suggest that interface roughness greatly decreases the importance of RISOP in Raman scattering from nonequilibrium phonons.

\footnotetext{
- Present address: Max-Planck-Institut fur Festkorperforschung, Stuttgart, Germany
} 


\section{Model caiculation and results}

Our model calculation is based on the model of electron-phonon (Fröhlich) interaction in $\mathrm{QW}$ proposed by Huang and $\mathrm{Zhu}^{2-4}$. The nonequilibrium phonon distribution $N_{q}$ is obtained by solving a set of rate equations similar to that described in Ref. 5. As can be seen from fig. 1(a), the nonequilibrium phonon distribution geinerated by intersubband scattering depends very strongly on the $\mathrm{QW}$ width. When the well width is around $185 \mathrm{~A}$ and the corresponding separation of the first two subbands is about $1 \mathrm{LO}$ phonon energy, a significant amount of small $q_{/ /}\left(<10^{5} \mathrm{~cm}^{-1}\right)$ phonons are generated by RISOP. This is shown more explicitly in fig. $1(b)$ where $N_{g}$ is plotted against well width for $q_{/ /}=7 \times 10^{4} \mathrm{~cm}^{-1}$.
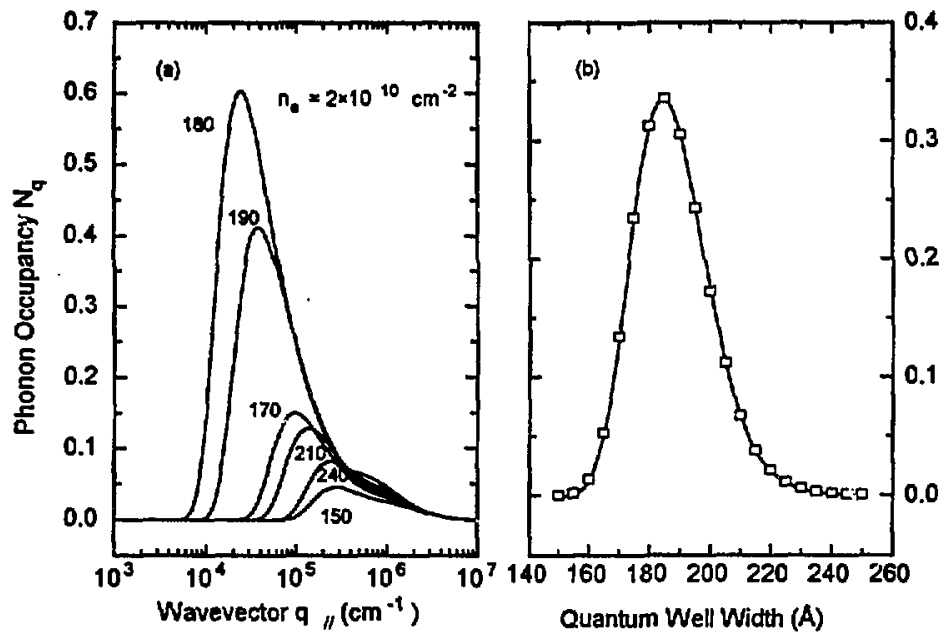

Figure 1. (a) Phonons generated by intersubband scattering as a function of in-plane wavevector $q_{/}$ calculated for an electron density of $2 \times 10^{10} \mathrm{~cm}^{-2}$ and for several QW widths (in $A$ ). (b) Occupancy of phonons with wavevector $a_{d / 3}=7 \times 10^{4} \mathrm{~cm}^{-1}$ as a function of well width. This wavevector corresponds to the actual experimental value.

\section{Experimental results and discussions}

Measurement of $N_{q}$ was performed with a 5 picosecond dye laser. The inelastically scattered light was dispersed by a Spex triple spectrometer and recorded by a multichannel detector. The $q_{/ /}$of phonons probed by Raman scattering is about $7 \times 10^{4} \mathrm{~cm}^{-1}$. Due to strong resonance Raman effect in QW, the usual way of determining $N_{q}$ by simply taking the ratio of Stokes $I_{S}\left(\omega_{\mathcal{L}}\right)$ and anti-Stokes intensity $I_{A S}\left(\omega_{\mathcal{L}}\right)$ at the same incident photon frequency $\omega_{L}$ is no longer valid. Loudon have shown that, in systems with time reversal symmetry, Stokes cross-section $\sigma_{\mathrm{S}}$ and anti-Stokes cross-section $\sigma_{\mathrm{AS}}$ satisfy the following relation: 6

$$
\sigma_{A S}\left(\omega_{L}-\omega_{L O}\right)=\sigma_{S}\left(\omega_{L}\right)
$$




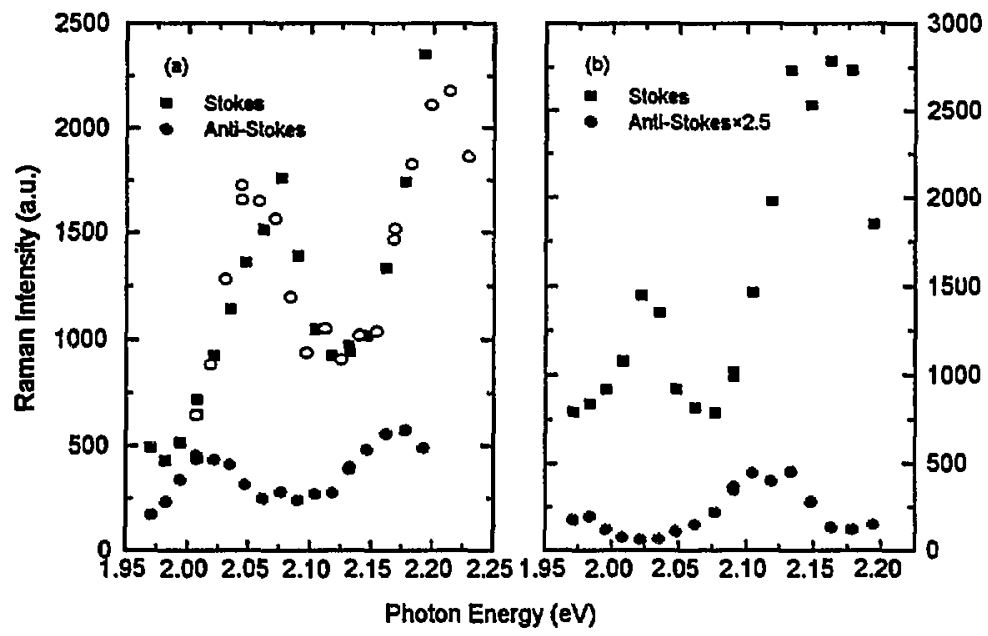

Figure 2. Dependence of Raman intensity on incident laser photon energy for a multiple QW sample with $190 \AA$ well width. (a) measurement at room temperature. Open circles represent anti-Stokes intensities after being multiplied by a factor of 3.8 and shifted to the right along the horizontal axis by 1 LO phonon energy, (b) measurement at liquid nitrogen temperature.

where $\omega_{L}$ is the LO phonon frequency. As a result the correct formula for determining the phonon occupancy is given by the following equation:

$$
N_{q}=\left[I_{S}\left(\omega_{L}\right) / I_{A S}\left(\omega_{L}-\omega_{L O}\right)-1\right]^{-1}
$$

To test the validity of eq. (1), we measured the Stokes and anti-Stokes Raman intensities in a QW sample at room temperature. As shown in fig. 2, the Stokes and antiStokes intensities show resonance peaks at different photon energies. However, their enhancement peaks are shifted by exactly $\hbar \omega_{L O}$ as predicted by eq. (1). This is shown in fig. 2(a) by multipling the anti-Stokes intensities by 3.8 and shifting the entire curve towards higher photon energy by $\hbar \omega_{L O}$. The $N_{q}$ calculated using eq. (2) is 0.35 and the corresponding phonon temperature is $330 \mathrm{~K}$. At liquid nitrogen temperature, anti-Stokes scattering became negligible unless the sample was excited by strongly focussed picosecond laser pulse. In this case the Raman signals were due entirely to scattering by nonequilibrium phonons. These showed similar enhancement peaks whose positions differ by $\hbar \omega_{10}$ between the Stokes and anti-Stokes spectra (see fig. 2(b)). The $N_{q}$ as determined from eq. (2) is shown as a function of well width in fig. 3 . In contrast to the results of the model calculation, there is no noticeable enhancement due to RISOP. In view of our experimental results, we propose the following explanation. We have assumed that $q_{/}$is conserved in Raman scattering. However, due to interface roughness, $q_{/ /}$may not be conserved exactly. Instead of probing phonons with a single value of $q_{/ /}$, we in fact measure phonons with a range of $q_{/ /}$. This range is approximately related to the average 
size $d$ of the atomically smooth regions as $2 \pi / d$ Recent experimental work on resonant acoustic phonon scattering have also found interface roughness to be important? ${ }^{7}$. These authors have found $d$ to be a few hundred $A$ in their samples. If we assume $d=100 \AA$ for our samples, we find qualitative agreement between our calculations and experimental results (fig. 3). This suggests that interface roughness play a very important role in our Raman scattering experiment.

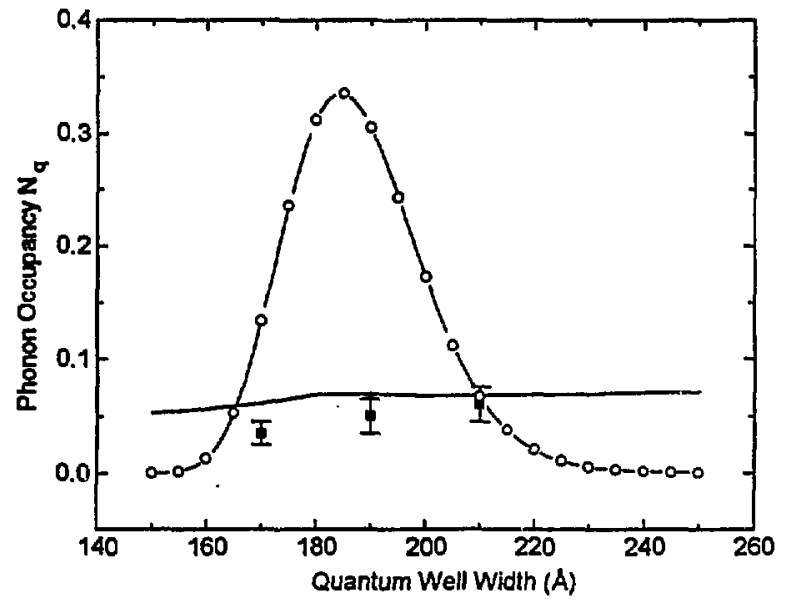

Figure 3. Nonequilibrium phonon occupancy vs. quantum well width for electron density of $2 \times 10^{10} \mathrm{~cm}^{-2}$, solid squares represent experimental data, solid line represents calculations including interface roughness effect, open circles sepresent calculations with conservation of $q_{/ / \text {. }}$

\section{Acknowledgments}

This work was supported by the Director, Office of Energy Research, Office of Basic Energy Sciences, Materials Sciences Division of the U.S. Department of Energy under Contract No. DE-AC03-76SF00098. TR acknowledges support of a IBM Postdoctoral Fellowship.

\section{References}

1. See, for example, S. Briggs and J. P. Leburton, Superlatt. Microstruct. 5, 145 (1989).

2. K. Huang and B. Zhu, Phys. Rev. B 38, 13377 (1988).

3. S. Rudin and T.L. Reinecke, Phys.Rev. B 41, 7713 (1990); B 43, 9298(E) (1991).

4. H. Rücker, E. Molinari, and P. Lugli, Phys. Rev. B 44, 3463 (1991).

5. K.T. Tsen, K.P. Wald, T. Ruf, P.Y. Yu, and H. Morkoç, Phys. Rev. Lett., 67, 2557 (1991).

6. R. Loudon, Proc. R. Soc. London A 275, 218 (1963).

7. T. Ruf, V.I. Belitsky, J. Spitzer, V.F. Sapega, M. Cardona, and K Ploog, Phys. Rev. Lett., 71, 3035 (1993). 